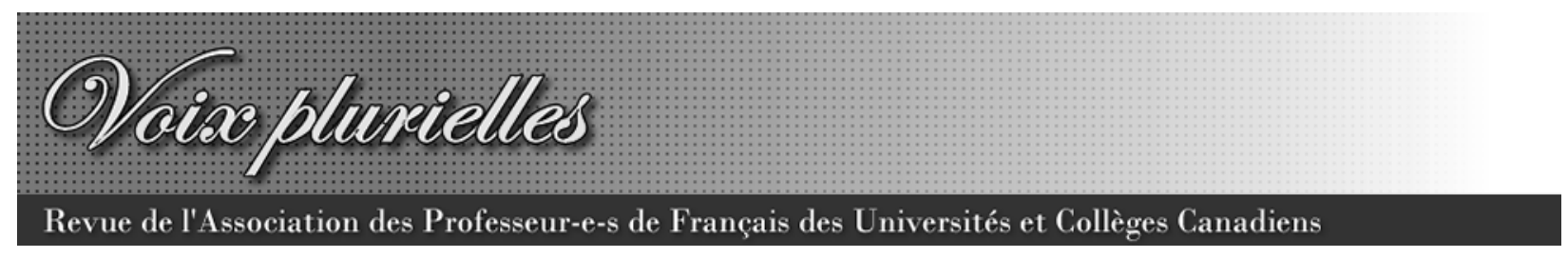

\title{
Voix plurielles
}

\section{Volume 1, Numéro 2 : février 2005}

\author{
Fevronia Novac
}

\section{Pavel, Thomas : La pensée du roman}

Citation MLA : Novac, Fevronia. «Pavel, Thomas. La pensée du roman.» Voix plurielles 1.2 (février 2005). 


\title{
Pavel, Thomas : La pensée du roman
}

\author{
Fevronia Novac \\ Université d'Ottawa
}

Février 2005

Thomas Pavel a l'ambition d'entreprendre une histoire du roman occidental de ses origines (qu'il situe dans le roman hellénistique) jusqu'à présent. Ses exemples étant circonscris aux romans qui font partie du canon littéraire occidental, le critique nous propose une « anthropologie » du roman élaborée en parallèle à une histoire des idées dans la tradition de l'histoire de la littérature universelle. Son étude se place loin des préoccupations contemporaines de remettre en cause les histoires littéraires du passé ou des plus récentes tentatives de réécrire et de ré-interpréter l'histoire du roman occidental, tentatives qui sont nombreuses dans le cadre de la critique littéraire américaine, à laquelle l'auteur, professeur à l'Université de Chicago, appartient.

En analysant les œuvres narratives qui ont été retenues comme fondamentales dans l'histoire littéraire de l'Ouest, Pavel se demande si ces œuvres sont «celles qui ont conquis la notoriété par leur nouveauté singulière (comme aurait aimé nous le faire croire le modernisme) ou celles qui, à l'inverse, ont incarné de manière exemplaire la pratique du genre à leur époque » (31).

Comme méthodologie, l'auteur emprunte sélectivement des démarches théoriques d'Aurebach, de Bakhtine et de Lukacs. Préoccupé par le dialogue entre les diverses époques dans l'histoire du roman, l'auteur se sert aussi, sans l'avouer, des conceptions de l'histoire littéraire de Hans Robert Jauss. Jauss est surtout un modèle pour Pavel lorsque le critique examine des œuvres nouvelles, sans frontière de langue (il discute des romans français, anglais, américains, russes, allemands, portugais, etc.), œuvres qui ont eu un impact sur la littérature à venir ou qui ont été choisies comme représentatives dans le canon de la littérature universelle. Or, pour Jauss, une œuvre nouvelle se définit en rapport avec toute une histoire littéraire du passé. Néanmoins, dans la conception de Jauss, cette œuvre acquiert d'autant plus de valeur qu'elle rompt avec la tradition. Cependant, Pavel ne semble pas partager cet avis car lui est plutôt à la recherche des similarités dans la pensée des œuvres qui connaissent des écarts historique et d'attente considérables. Le plus souvent, dans l'étude de Pavel, les œuvres nouvelles s'expliquent par celles qui les ont précédées.

Pour Pavel, l'histoire du roman occidental est autant un parcours vers l'invention de nouvelles formules qu'une progression logique basée sur les traditions normatives du passé. Selon le critique, la spécificité du roman réside dans sa manière de penser la relation du personnage à son idéal (manière qui diffère en fonction de la période historique dont le roman est le résultat). La pensée d'une époque rend possible, dans la vision de Pavel, la compréhension de la «matière anecdotique 
»(46) des romans qui sont issus de la période historique respective. Pour simplifier, l'anthropologie du roman proposée par l'auteur pose la « question axiologique » suivante : « pour défendre l'idéal, l'homme doit résister au monde, s'y plonger pour y rétablir l'ordre moral ou enfin s'efforcer de remédier à sa propre fragilité, si, en d'autres termes, l'individu peut habiter le monde où il voit le jour » (47).

C'est dans la pensée de cette " anthropologie fondamentale » de l'interaction du personnage avec son milieu et son idéal moral que Pavel découvre une constante dans l'évolution du roman et c'est cette réflexion qui simplifie sa tâche, qui autrement pourrait paraître impossible à achever.

Pavel débute son histoire du roman avec une discussion du roman hellénistique où l'idéal moral des personnages est accompli grâce à l'intervention divine. L'auteur étudie les paradigmes successifs dans l'histoire du roman universel comme réponse à la « question axiologique posée dès ses origines par le roman » (209) à l'égard de l'idéal moral dans sa relation avec le monde.

Dans le roman de l'époque prémoderne au XVIIIe siècle, l'idéal se trouve dans le cœur de l'homme (141). Pavel regroupe sous le titre «L'enchantement de l'intériorité » une vaste gamme des romans, du roman sentimental au roman gothique, de l'idéalisme romanesque au scepticisme. Goethe (Les souffrances du jeune Werther) est un des écrivains les plus complexes de cette époque car, chez lui, « l'exaltation de l'amour » ne mène pas à la réconciliation de l'âme avec le monde qui l'entoure (188). Par la suite, Pavel place le réalisme et le naturalisme dans le cadre de l'antiidéalisme romanesque car ces courants littéraires montrent leur scepticisme envers " la possibilité de la beauté morale individuelle » (290-291).

$\mathrm{Au}$ XIXe siècle, l'idéalisme moderne se soucie de « concilier la représentation de l'âme héroïque avec celle de la vraisemblance du monde » (217). Dans ce sens, Walter Scott marque un tournant dans l'histoire du roman. Avec lui, « la pensée du roman passe de l'anthropologie générale, fondée sur des considérations normatives universelles, à l'anthropologie comparative, dont l'objet est la diversité des liens normatifs établis par les communautés humaines » (228).

L'auteur se préoccupe même des considérations de style lorsqu'il explique l'origine du discours indirect libre par le contexte historique où, «d'une part, le for intérieur des êtres humains est reconnu, grâce à l'enchantement de l'intériorité, comme la source de leur dignité morale, et où, d'autre part, grâce à la critique de l'idéalisme romanesque, cette dignité ne se laisse pas assimilée naïvement avec la perfection » (272). Pavel analyse le discours indirect libre chez Jane Austen, écrivaine « empathique » qui semble être une de ses auteures préférées dont les découvertes en matière de psychologie du personnage ouvriront la voie du roman d'analyse pratiqué par Henry James, Wilhelm Raabe et Joseph Conrad (273).

Le critique considère l'évolution du roman comme un dialogue constant avec les formules précédente du genre. Les grands axes de son histoire littéraire semblent posés par les romans hellénistique et prémodernes, romans auxquels Pavel revient tout au long de son parcours historique.

Pavel ne voit pas de coupure radicale avec la tradition dans l'évolution du genre romanesque. Au contraire, il perçoit des dialogues entre les époques, de l'intertextualité et des synthèses. Selon lui, " à la fin du XVIIIe siècle, le roman a achevé [...] la conversion de l'idéalisme ancien, fondé sur l'alliance entre le héros ou le couple solitaire, et la norme transcendante garantie par la Providence, à l'idéalisme moderne, qui célèbre l'énergie normative et l'autosuffisance de la belle 
âme » (207).

Le XXe siècle est moins présent dans l'étude de Pavel. Le critique divise les romans contemporains entre les « difficiles » (qu'il choisit de ne pas discuter) et les « accessibles » qu'il organise dans quatre catégories : moralistes, romans d'analyse sociale, néo-romantiques et « les héritiers de la tradition comique et sceptique » (397). Lorsque l'auteur se penche sur le roman du XXe siècle, c'est pour examiner l'esthétisme qui, selon lui, ouvre la voie du modernisme. En élaborant sur la fonction contradictoire de l'esthétisme, Pavel oppose Proust à Joyce : « L'art, qui chez Proust libérait l'homme de sa servitude, sert ici [chez Joyce] à la mettre en lumière et non pas à la vaincre » (374). Conformément à sa trajectoire initiale de pensée, Pavel découvre que les romans modernistes marquent, eux aussi, un retour à l'anthropologie des romans hellénistiques et baroques (388) et que Gabriel Garcia Marquez, le postmoderniste, réintègre « les techniques du roman prémoderne et populaire » (394). Pavel justifie l'absence de la littérature du XXe siècle dans son étude par un besoin de recul historique pour mieux discerner la pensée qui informe des œuvres plus récentes (401).

Le critique se concentre sur les tournants dans l'histoire du roman universel et dont il isole plusieurs moments mémorables : d'une part, les œuvres qui contribuent à un changement radical de paradigme dans la pensée du roman et, d'autre part, les « chefs d'œuvre » tels Don Quichotte, Tom Jones, À la recherche du temps perdu (410). L'étude de Pavel n'est pas philosophique ; l'auteur se contente d'observer l'anthropologie romanesque sans élaborer jusqu'à théoriser la pensée d'une époque à la manière de Foucault.

Même si son approche pourrait sembler linéaire, Pavel comparant les romans de différentes époques à partir de l'anthropologie fondamentale définie, dans ses grandes lignes, par le roman hellénistique, l'auteur admet le besoin de l'historien du roman de scruter la multiplicité des thèmes romanesques dans leur diversité (409-410). Son travail d'érudition sera utile à tous ceux intéressés par une lecture cohérente des grandes lignes de l'histoire du roman universel. 